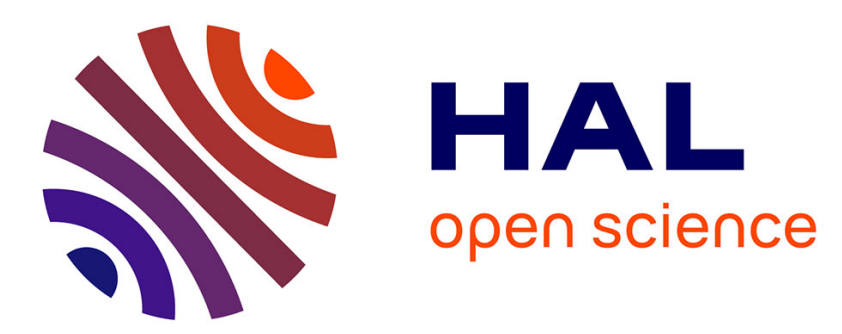

\title{
Energy-aware Cache Placement Scheme for IoT-based ICN Networks
}

Oussama Serhane, Khadidja Yahyaoui, Boubakr Nour, Hassine Moungla

\section{To cite this version:}

Oussama Serhane, Khadidja Yahyaoui, Boubakr Nour, Hassine Moungla. Energy-aware Cache Placement Scheme for IoT-based ICN Networks. ICC 2021: IEEE International Conference on Communications, Jun 2021, Montreal (virtual), Canada. pp.1-6, 10.1109/ICC42927.2021.9500341 . hal03161227

\section{HAL Id: hal-03161227 \\ https://hal.science/hal-03161227}

Submitted on 5 Mar 2021

HAL is a multi-disciplinary open access archive for the deposit and dissemination of scientific research documents, whether they are published or not. The documents may come from teaching and research institutions in France or abroad, or from public or private research centers.
L'archive ouverte pluridisciplinaire HAL, est destinée au dépôt et à la diffusion de documents scientifiques de niveau recherche, publiés ou non, émanant des établissements d'enseignement et de recherche français ou étrangers, des laboratoires publics ou privés. 


\title{
Energy-aware Cache Placement Scheme for IoT-based ICN Networks
}

\author{
Oussama Serhane*, Khadidja Yahyaoui*, Boubakr Nour ${ }^{\S}$, and Hassine Moungla ${ }^{\ddagger}$ \\ ${ }^{*}$ Computer Science Department, Université Mustapha Stambouli, Mascara, Algeria \\ ${ }^{\S}$ School of Computer Science, Beijing Institute of Technology, Beijing, China \\ $\ddagger$ Université de Paris, LIPADE, F-75006 Paris, France \\ IIUMR 5157 CNRS, Telecom SudParis, Institut Polytechnique de Paris, Palaiseau 91120, France \\ Email: \{oussama.serhane, khadidja.yahyaoui\}@univ-mascara.dz,n.boubakr@bit.edu.cn, hassine.moungla@u-paris.fr
}

\begin{abstract}
The Internet of Things (IoT) is overrunning different domains and applications, where the use of wireless sensors and mobile devices is indispensable in such a mobile environment. These heterogeneous devices may generate a tremendous amount of content. Information-Centric Network (ICN) paradigm has been proposed to meet today's users and application requirements. The in-network caching is a fundamental feature supported by design in ICN that improves network performance by providing ubiquitous caching in the network layer. Since most IoT devices are resource-constrained with limitations in communication, processing, energy, and memory; the energy-efficiency is a prime concern in IoT deployment. Different factors may affect energy efficiency in ICN-based wireless IoT networks such as transport (communication), caching, and energy limitation. This research paper attempts to focus on the in-network caching in wireless IoT to maximize the energy-efficiency. We propose an Energy-aware caching placement scheme (EaCP) that aims to maximize the energy-saving by trading-off between content transmission energy and content caching energy. Compared to other strategies, the simulation results show significant improvements while ensuring low data replication and a high cache hit ratio.
\end{abstract}

Index Terms-Information-Centric Networking (ICN), Internet of Things (IoT), In-network caching, Energy efficient

\section{INTRODUCTION}

The Internet of Things (IoT) is uniting its integration with different domains and applications, including smart cities, smart homes, smart transportation, etc. It is witnessing an exponential growth of devices that may connect in a wired or wireless communication mode. Most IoT devices are considered as resource-constrained with limitations in processing, memory, and energy [1]. Thus, different operating systems and protocols have been proposed to enable smart services in such a constrained environment.

The traditional host-centric model does not, at least in part, fulfil the requirements of today's Internet applications and user demands [2]. This model has been refined to be adapted with the current complex Internet system where security, mobility, and flexibility are challenging requirements. Furthermore, Information-Centric Networking (ICN) [3] has become one of the hot topics for future Internet that may replace the current host-centric model. ICN uses the content name as the fundamental element instead of host address [4]. Hence, name-based routing rules are applied to discover the content and deliver it back to requesters. Moreover, ICN incorporates content-based security concept by integrating all securityrelated information with the content itself [5]. Besides, the transparent in-network caching is considered as one of the main ICN features that may speed up the content retrieval from the closer cache-store and improve the resource utilization [6].

Various efforts have been presented in the literature to use ICN on top of IoT [7]. For instance, work in [8] proposes a distributed ICN architecture for eHealth applications. The proposed architecture can deal with communication models, mobility, and management. Work in [9] focuses on Quality of Service (QoS) provisioning in IoT by supporting both Push and Pull traffic. The proposed algorithm sorts and classifies IoT traffic to query-based, time-based, or event-based, and incorporates a traffic-based caching concept. Work in [10] proposes a multi-metric robust interface ranking scheme to improve QoS performance.

Despite the existing efforts, the explosive increase of IoT devices and the use of ICN as a communication-enabler for IoT applications open new challenges for the energy efficiency communication where the later is a growing concern. Work in [11] surveys the content placement, caching, and routing techniques from energy-efficient perspective. However, the authors did not give much importance to IoT applications/characteristic in their study.

The motivation behind this work is to propose an ICN caching scheme for wireless IoT taking energy efficiency as the main factor. Hence, we model the content placement problem in wireless IoT networks as an integer combinatorial problem and propose an energy-aware heuristic algorithm to decide the optimal cache placement with the objective of maximizing the overall energy efficiency. The extensive simulation shows that our proposed scheme records a noteworthy energysaving with low data replication while increasing the cache hit ratio.

The rest of the paper is organized as follows. Section II presents related work. Section III defines the system model and problem formulation. Section IV details the proposed scheme. Section V shows the performance evaluation and discusses the obtained results. Section VI concludes the paper and highlights future works. 


\section{RELATED WORKS}

To date, excellent research results have been shown in ICN, including in-network caching [12] as well as ICN-IoT [13]. A recent draft by Information-Centric Networking Research Group (ICNRG) [14] presents a mechanism that aims to discover the information about the network topology as well as the path and content caching in Content-Centric Networks (CCN). Meddeb et al. [15] present a qualitative and quantitative comparison on the cache placement and replacement in NDN networks. To determine the caching performance, the authors simulated different scenarios. However, the used scenarios are general in nature and may not be suitable for IoT applications.

Focusing on energy efficiency, Zou et al. [16] present a lightweight probabilistic forwarding strategy aiming at enhancing the energy in resource-constrained devices. The authors include the node's Media Access Control (MAC) address in packets. Thus, the broadcast is used for interest forwarding, and unicast for data forwarding. Although a probability function has been used to enhance the energy during the forwarding, the use of MAC addresses brings ICN paradigm back to the host-centric model, which may violate the contentcentric goal. Gao et al. [17] propose a dual-mode interest forwarding scheme in Named-Data Networks (NDN). The proposed scheme consists of combining the forwarding plane with mechanisms (e.g., flexible mode shift, flooding control, and broadcast storm avoidance), thus enhancing energy consumption. However, the execution of such mechanisms may consume more energy. Jsm et al. [18] design a new forwarding strategy in order to reduce energy consumption in an IoT network. The authors also modify the naming structure by adding the name of the application generating the request to the interest packet. However, the proposed scheme limits the scalability of the network by increasing the load at the gateway. Wang et al. [19] propose a QoS routing mechanism taking the energy-efficient as a fundamental factor. The mechanism consists of evaluating link-state suitability to reply to users' QoS requirements and energy-aware link monitoring based on traffic. A color management strategy is used to coloring the outgoing interface and avoid failed interest packet issues. Hahm et al. [20] study the existing distributed caching strategies for IoT content in order to capture most of the event observed by IoT devices and enable these devices to be in sleep mode in order to save energy. However, the authors present preliminary experimental results in small networks.

To expand on the point, other caching strategies [21] are widely used in ICN, such as Leave Copy Everywhere (LCE), Leave Copy Down (LCD), Edge Caching (EC), and Consumer Cache (CC). In LCE, all involved nodes in the communication path have to keep a copy of the content. This scheme's main drawback is the same content may be cached multiple times in the path (duplicated data). Hence the cache diversity is shallow. LCD caches the content only one-hop after the producer. It reduces communication only one-hop. Similarly, EC caches the content at the edge node of the network,
TABLE I: Notations used in the paper.

\begin{tabular}{ll}
\hline & \multicolumn{1}{c}{ Parameters of the Model } \\
\hline $\mathcal{N}$ & Set of nodes \\
$A T$ & Set of Access Things \\
$E T$ & Set of Edge Things \\
$\mathcal{R}$ & Set of routers with caching capabilities \\
$\mathcal{L}$ & $\mathcal{L}=\{\mathcal{R} \cup E T\}$ Set of replica-nodes \\
$\mathcal{F}$ & Content catalogue \\
$\mathcal{T}_{l}$ & Number of content stored in the cache-store $l$ \\
$\mathcal{S}_{f}$ & Content size \\
$\mathcal{E}_{i, l, f}$ & Overall energy required to fetch $f$. \\
$\mathcal{E}_{i, l, f}^{c}$ & Energy required to cache $f$ in $l$ \\
$\omega_{c}$ & Required energy by the hardware. \\
$C_{\max , l}$ & The maximum cache capacity of the node $l$. \\
$Q_{\max }$ & QoS assignments or link transfer capacity. \\
$\mathcal{D}_{i, l, f}$ & Data rate to fetch content from cache store $l$. \\
$\mathcal{D}_{i, l, f}$ & Data rate to fetch content from original producer $p$. \\
$k_{l}^{f}$ & $\{0-1\}$ Cache storage reachability: \\
& $k_{l}^{f}=1$ if replica node $l \in L$ can serve file $f \in F$ \\
$x_{l}^{f}$ & $\{0-1\}$ Cache decision variable : \\
& $x_{l}^{f}=1$ if node $l \in L$ decides to keep the content $f \in F$ \\
\hline
\end{tabular}

closer to the end-users. Although EC improves the network performance and content retrieval, deciding which content to cache in the edge node remains an open challenge. Finally, $\mathrm{CC}$ caches the content one-hop after the consumer regardless of the number of the consumer(s), which means it may end up caching non-popular content in non-optimal places.

\section{System Model \& PROBlem Formulation}

\section{A. System Model}

In order to design an efficient IoT network energy model, we split the model into two essential areas: the network model and the energy model.

1) Network Model: Fig. 1 illustrates a scenario of caching system model for wireless IoT. An IoT network is represented as a graph $\mathcal{G}=(\mathcal{N}, \mathcal{A})$, where $\mathcal{N}$ represents a set of nodes, and $\mathcal{A}$ set of links (wired and wireless). The network is modeled as a multi-hop model, where nodes can be classified into: Access Things $(A T)$, Edge Things $(E T)$, and intermediate routers $(\mathcal{R})$ with the caching capabilities. ATs represent IoT users, while $E T$ s are the edge devices. We assume that $\mathcal{R}$ s are randomly placed in the network. A set of $A T$ s connected to the same ET is named IoT-Net.

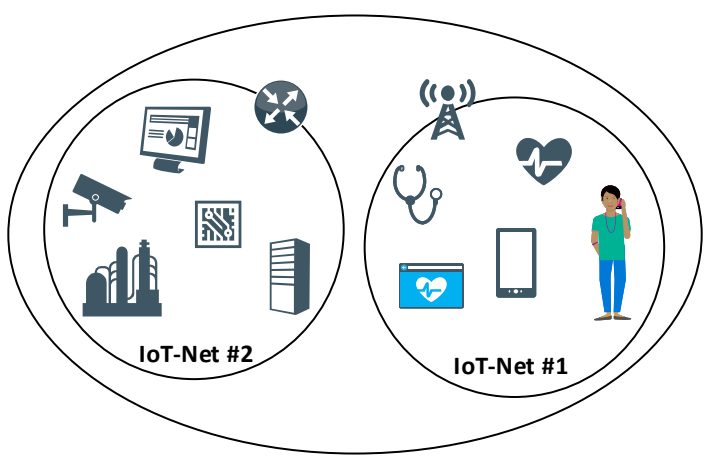

Fig. 1: Caching System Model for Wireless IoT. 
We define $\mathcal{L}=\{\mathcal{R} \cup E T\}$ as a set of nodes who can cache and offer contents (replica-nodes), and $\mathcal{F}$ content catalogue. We assume that content chunks have the same size. Each $l \in \mathcal{L}$ has a finite cache-size $C_{\max }$, in which the occupied size is defined by $\mathcal{T}_{l \in \mathcal{L}} \times \mathcal{S}_{f \in \mathcal{F}}$, where $\mathcal{T}_{l}$ denotes the number of contents stored in the cache-store, and $\mathcal{S}_{f}$ denotes the content size. Moreover, each link $a_{l} \in A$ is restricted with max capacity $Q_{\max }$ according to the link transfer capacity or available QoS assignment.

2) Energy Consumption Model: In IoT network, each user $i \in \mathcal{A T}$ might be assigned at least to one $\mathcal{E} \mathcal{T}$. User request Req may be satisfied by any replica node $l$ in the IoT-Net or the original producer $p$.

Two influence elements impact the energy consumption in the IoT network: the content transmission energy: to request and deliver the content, and the content caching process: to cache the content at the local node.

Content Transmission Energy. The content transmission energy varies depending on the content availability place: (a) the content is satisfied in the network by any cache-store, (b) the request reaches the original producer where the content is delivered back to the requester. In doing so, we denote $k_{l}^{d}$ as the content storage reachability.

$$
k_{l}^{f}= \begin{cases}1 & \text { if file } f \text { is served by cache-store } l . \\ 0 & \text { else. }\end{cases}
$$

When the content is satisfied by a cache-store $l \in \mathcal{L}$, we define the data rate by:

$$
\mathcal{D}_{i, l, f}=\log _{2}(1+\operatorname{SINR}(i, l, f))
$$

where $\operatorname{SINR}(i, l, f)$ describes the signal to interference noise ration.

Otherwise, the request must be forwarded to fetch the content from the original producer $p$. The energy consumption in such a case is defined as:

$$
\mathcal{D}_{i, p, f}=\log _{2}(1+\operatorname{SINR}(i, p, f))
$$

where $\operatorname{SINR}(i, p, f)$ describes the signal to interference noise ratio in the system. Furthermore, as long as content delivered is imporrant, we define $\mathcal{T}^{\mathcal{T}}$ as the total throughput to fetch content $f \in F$ requested by the user $i \in A T$ over the network by:

$$
\mathcal{T}^{\mathcal{T}}=\left(k_{l}^{f} \times \mathcal{D}_{i, l, f}\right)+\left[\left(1-k_{l}^{f}\right) \times \mathcal{D}_{i, p, f}\right]
$$

Content Caching Energy. Let $\mathcal{E}_{i, l, f}^{c}$ denote the required energy to cache content $f$ in node $l$. The cache energy consumption $\mathcal{E}_{i, l, f}^{c}$ can also be defined based on the occupied cache size, as below:

$$
\mathcal{E}_{i, l, f}^{c}=\mathcal{S}_{f} \times \omega_{c}
$$

where $\omega_{c}$ denotes the required energy by the hardware in watt/bits, while $\mathcal{S}_{f}$ defines the occupied cache size. We also denote $x_{l}^{f}$ as the cache decision variable:

$$
x_{l}^{f}= \begin{cases}1 & \text { if node } l \text { decides to cache the content } f . \\ 0 & \text { else. }\end{cases}
$$

Hence, let $\mathcal{T}^{\mathcal{E}}$ represent the caching energy required as follow:

$$
\mathcal{T}^{\mathcal{E}}=x_{i}^{f} \mathcal{E}_{i, l, f}^{c}
$$

Global Energy Consumption. Finally, the total IoT-Net energy consumption can be calculated as follow:

$$
\mathcal{E} \mathcal{E}=\sum_{l \in \mathcal{L}} \sum_{f \in \mathcal{F}} \mathcal{T}^{\mathcal{T}}+\mathcal{T}^{\mathcal{E}}
$$

\section{B. Problem Formulation}

From the ICN perspective, the total consumed energy is defined by the sum of both transport energy and cache energy. The former includes the energy consumption to forward the content from the original producer to the cache-store(s) as well as from the cache-store(s) to the consumer(s). The latter relies on the wasted energy to cache the content, and it depends on hardware technology (e.g., SSD, TCAM, DRAM, etc).

Our concern is then to extend energy efficiency by minimizing the total consumed energy, which is described as follow:

$$
\min \mathcal{E} \mathcal{E}
$$

Subject to:

$$
\begin{gathered}
\sum_{i \in A T} k_{l}^{f} \mathcal{E}_{i, l, f} \leq Q_{\text {max }}, \quad \forall l \in \mathcal{L} \\
\sum_{l \in \mathcal{L}} k_{l}^{f} \mathcal{D}_{i, l, f} \geq \mathcal{D}_{t h}, \quad \forall i \in A T \\
\sum_{f \in \mathcal{F}} x_{l}^{f} \mathcal{T}_{l} \mathcal{S}_{f} \leq \mathcal{C}_{\text {max }}, \quad \forall l \in \mathcal{L} \\
\sum_{e=1}^{|E T|} k_{l}^{f} \leq M, \quad \forall f \in \mathcal{F} \\
x_{l}^{f} \in\{0,1\}, \quad \forall l \in \mathcal{L} \\
k_{l}^{f} \in\{0,1\}, \quad \forall l \in \mathcal{L}, \forall f \in \mathcal{F}
\end{gathered}
$$

Eq. (9) represents the global objective function that aims to minimize the overall energy consumption of the network. Constraint (10) indicates that the energy to fetch the content $f$ should be bounded by the maximum defined transmission energy $Q_{\max }$ (either from wired or wireless transmission capacity or defined QoS border). While constraint (11) represents that the data rate to cache a file $f$ should be greater than the threshold $\mathcal{D}_{t h}$. Constraint (12) indicates that the cache storage must not go beyond the maximum storage capacity. Similarly, constraint (13) indicates that a file $f$ can be cached at maximum on $M$ cache stores. Finally, constraints (14) and (15) imposes the non negativity on node caching decision and cache storage reachability, respectively.

\section{EACP: EnERgy-AWARe CAChe Placement Scheme}

The high diversity of real-world content embedded in the IoT networks requires considering different factors that influence and impact energy saving while ensuring a high energy efficiency level. In this section, we describe the proposed caching strategy, namely EaCP. 


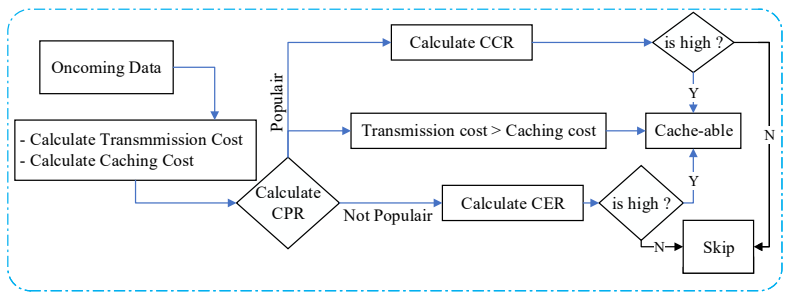

Fig. 2: EaCP working mechanism.

1) Basic Metrics: In this part, we highlight the basic metrics used by EaCP.

- Content Popularity Ratio (CPR): In IoT networks, content popularity varies over time. Thus, let $\phi_{i}^{f}$ denote the popularity value of content $f$ at node $i$. The CPR is obtained by dividing the received requests for content $f$, by all received requests at the node $i$, in a defined time period, which described as follow:

$$
\phi_{i}^{f}=\frac{\text { Number of received requests for } f}{\text { Total of all received requests }}
$$

- User Proximity Ratio (UPR): This value indicates the node's position in the network. UPR outlines the relationship linking the content size, node degree (number of neighbors requesting the content), and the sum of the arrival demands, which is described in the following equation:

$$
v_{i}^{f}=\frac{i^{\partial}}{\sqrt{\varsigma_{r}^{i} \cdot \varsigma_{r}^{p}}}
$$

- Content Caching Reward (CCR): This value is used to extract the reward value relative to client satisfaction to keep the content cached. CCR aims at making a balance among the content cache size, user satisfaction, and node importance, as described in the following equation:

$$
\varpi_{i}^{f}=\sqrt{\frac{i^{\partial} * \phi_{i}^{f}}{\mathcal{S}_{f} \cdot \phi_{i}^{f}}}
$$

$\varpi$ denotes the content caching reward, and $i^{\partial}$ denotes the node's neighborhood degree.

- Content Energy Reward (CER): This metric aims to generate the reward energy value of content in the case of a caching decision. As shown in Eq. 19, CER tends to balance the content fetching energy, the caching energy, and user demand satisfaction by prioritizing the most requested content.

$$
\psi_{i}^{f}=1-e^{\frac{\mathcal{T}^{\mathcal{T} * \phi_{i}^{f}}}{\mathcal{T}^{\mathcal{E}}}}
$$

2) Working Mechanism: In this part, we discuss the fundamental pieces of EaCP scheme. For simplicity purposes, we divide its working mechanism into multiple steps, as depicted in Fig. 2 and discussed below.

- Step 1. The process starts by collecting the node-local information, including the content-cache capacity cost, received demands for the content, and node importance. Then, it obtains the neighbor-nodes-local information (neighbors for each received request for the candidate content).

- Step 2. It aims to compute both of the required transmission energy to fetch the content from the cache-store $K$ or the original producer $P$, and calculate the required energy to cache content as represented in Eq. (4), and Eq. (7), respectively.

- Step 3. The process starts by calculating the popularity ratio (this mechanism aims to specify if the content is popular or not by comparing the CPR value to the defined threshold at the concerned node), then matches the content in multiple possible outputs in order to handle them on the next phase.

- Step 4. It tends to treat the different cases related to the caching decision. The process starts by marking if the content is cache-able or not based on different factors.

- Case 1: If the fetching energy is higher than the caching energy and the content is popular, then mark the content as cache-able. The node with the maximum UPR is cached.

- Case 2: If the content is not popular and the transmission energy is low than the caching energy, then calculate the content caching reward. The node with the maximum CCR is cached.

- Case 3: If the content is not popular and the transmission energy is high than the caching energy, we use the energy reward to decide the content caching where the node with maximum CER is selected.

- Case 4: If the content is not popular and the caching energy is high than the fetching energy, we do not cache the content.

Due to the massive amount of content generated by IoT devices, the EaCP scheme fits on multi-stage filtering rules, where the simplicity behind the EaCP mechanism aims to make fluent and optimal cache decisions with low complexity and overhead.

\section{Performance Evaluation}

In this section, we describe the performance evaluation of the EaCP caching scheme against the most used caching strategies.

\section{A. Simulation Setup}

We have implemented the EaCP scheme using the Python programming language. We have used the Barabasi network as a network core that connects with multiple groups of IoTs networks. The distribution of IoT nodes among these networks follows Normal Distribution low. We have also used Zipf distribution, which is suitable for this scenario, to model content requests' density. Table II provides a summary of the simulation setup as well as the used parameters. 
TABLE II: Simulation Parameters.

\begin{tabular}{ll}
\hline Parameter & Value \\
\hline Size of content & {$[512$ Byte, 9 Kilobyte $]$} \\
Number of unique contents & {$[100,1000]$} \\
Zipf parameter $(\alpha)$ & {$[1.1,1.5]$} \\
Number of node & {$[20,600]$} \\
Number of IoT-Net & {$[4,40]$} \\
Memory power efficiency & $6.25 \times 10^{-12}(\mathrm{watt} / \mathrm{bit})$ \\
Transport equipment (Core Nodes) & $1.7 \times 10^{-8}(\mathrm{Joule} / \mathrm{bit})$ \\
Transport equipment (Gateway nodes) & $1.38 \times 10^{-7}(\mathrm{Joule} / \mathrm{bit})$ \\
Transport equipment (Links) & $5 \times 10^{-9}($ Joule/bit) \\
Transport equipment (Edge Nodes) & $2.63 \times 10^{-8}($ Joule/bit) \\
\hline
\end{tabular}

\section{B. Evaluation Metrics}

- Cache Utilization: measures how many times the same content has been duplicated in the network's cache-store.

- Cache Hit Ratio: measures how many times a received request has been satisfied by the local node's cache-store than the overall received requests.

- Energy Saving: measures the saved energy by calculating the difference between the energy consumption if the content is served from the original producer or a replica cache-store.

- Energy Efficiency: shows the percentage of the saved energy compared to the consumed energy during the cache replacement process.

\section{Results and Discussion}

To show the proposed strategy's efficiency, we have benchmarked EaCP against popular caching schemes in ICN. We have also conducted simulations considering different aspects such as memory usage and energy efficiency. In doing so, we first started with a fixed content size and then extended it to a large-scale scenario.

Identical Content Size Experiment. Fig. 3 shows the cache utilization performance when the number of IoT nodes increases. We can observe the number of data copies constantly increasing in each of the strategies. Since LCD caches the content only on one node after the producer, we can notice that the cache utilization increases slightly. Similarly, EC has more extensive performance but imperceptibly different since we might have multiple edge nodes (based on the network

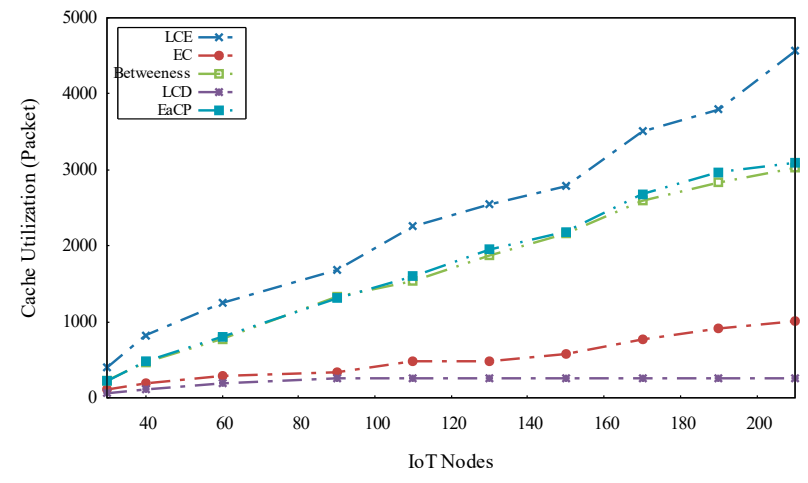

Fig. 3: Cache Utilization.

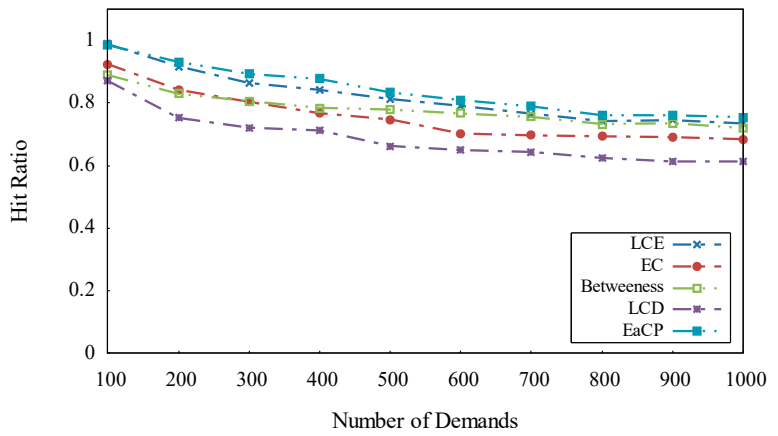

Fig. 4: Cache Hit Ratio.

topology). Additionally, LCE has the worse cache utilization since it caches content (high duplicated copies) along the communication path. Meanwhile, $\mathrm{EaCP}$ and Betweeness have similar output since they select only nodes with the optimal placement. This is because of the identical content size used in this experiment where it is an essential metric in deciding the caching energy reward in EaCP.

Fig. 4 depicts the average of the cache hit ratio when the number of requests increases. Both LCD and EC show a lower hit ratio performance due to the small number of involved nodes to cache content. Betweenness and LCE present considerable improvement. Besides that, $\mathrm{EaCP}$ shows better hit ratio improvements.

Large-scale Network with Content Size Variation. In order to study the performance of the proposed scheme in a large-scale network, we have increased the number of nodes and used a content size variation instead of identical size. Here, the prime objective is to show the caching scheme's real performance in terms of energy usage and energy efficiency. Fig. 5 shows the energy-saving ratio against the rising of content size. We observe that the energy-saving varies for each data size value. EC and LCD record the lowest energy saving among the rest of the strategies. Along with it, we observe that for the first content size LCE, Betwennes and EaCP show almost a little saving energy value and this is due to the small content size that improves the content available at the network.

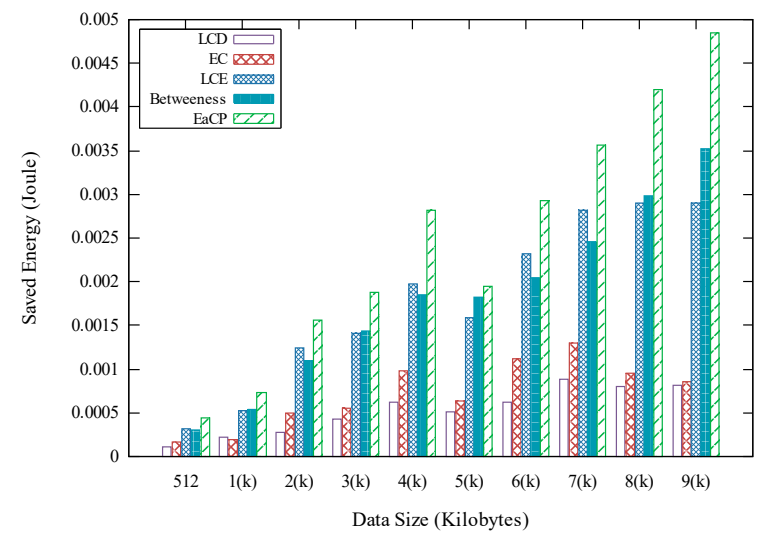

Fig. 5: Energy Saving. 


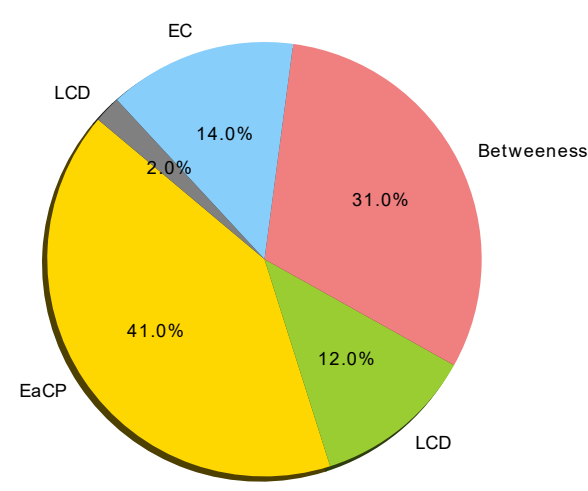

Fig. 6: Energy Efficiency.

However, when the content size starts increasing, EaCP gives a high rise in energy saving.

Fig. 6 shows the obtained results for energy efficiency. We observe that LCE shows almost non-existent energy efficiency. Indeed, it performs a large replacement execution, which diminishes its efficiency. EC and LCD use a limited number of nodes that reflect to limited energy consumption. Furthermore, Betwennes shows considerable efficiency, while EaCP is more efficient and outperforms the other strategies. This proves that $\mathrm{EaCP}$ has a notable achievement as it is able to save energy with low overhead.

Fig. 7 depicts the cache utilization against the rising of data size in a fixed number of network nodes along with the increase of the content size. Due to the cache-store's limited memory size, we observe a continuous decline in the data replication for all of the strategies. More specifically, we notice that LCD and EC start with low data replication and diminishing until stabilizing. In this scenario, EaCP shows a low data replication compared to LCE and Betweenness and reviles a wise memory usage.

\section{CONClusion AND Future Work}

In this work, we proposed EaCP strategy in order to improve the energy efficiency of IoT-based ICN networks. EaCP tends to balance the caching cost and the transmission cost in order to achieve a better content placement position. To doing so, we considered content transmission and caching energy, the node position, and content popularity. EaCP algorithm aims to achieve the optimal placement setting in a distributed

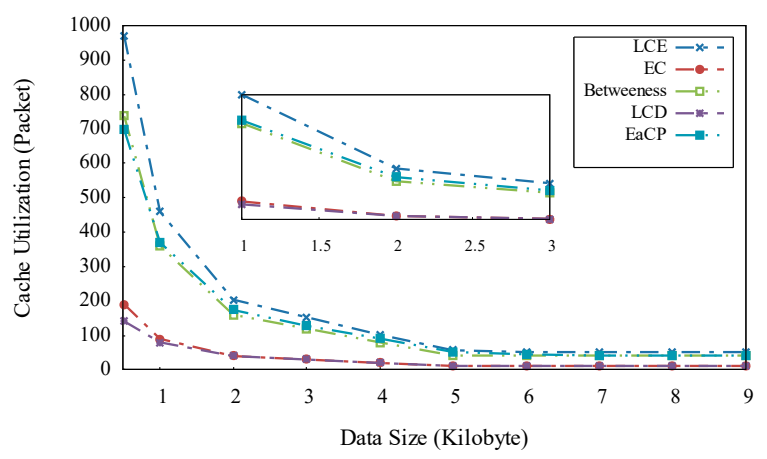

Fig. 7: Cache utilization per data size. fashion. In addition, we conducted an in-depth simulation considering various metrics. The obtained results proved the effectiveness of our schemes in terms of energy-saving, cache utilization, and considerable improvement in the cache hit ratio. We believe that the $\mathrm{EaCP}$ scheme is able to extend further the battery lives for IoT devices while strongly reduce the communication overhead. In the next phase of this work, we tend to improve the $\mathrm{EaCP}$ framework by concentrating on adapting real-world scenarios such as mobility. In such a situation, a smart deep learning model can be adopted to predict and extract the energy of IoT users' rewards for mobile nodes.

\section{ACKNOWLEDGMENTS}

This work is supported by the Ministry of Higher Education and Scientific Research of Algeria (MESRS) under Grant No. CO0L07UN290120190001. Dr. Yahyaoui is corresponding author.

\section{REFERENCES}

[1] M. J. Ali et al., "Interference avoidance algorithm (IAA) for multi-hop wireless body area network communication," in HealthCom Conference. IEEE, 2015, pp. 540-545.

[2] B. Nour et al., "ICN publisher-subscriber models: Challenges and groupbased communication," IEEE Network, vol. 33, no. 6, pp. 156-163, 2019.

[3] B. Nour et al., "NNCP: A Named Data Network Control Protocol for IoT Applications," in IEEE CSCN Conference, 2018.

[4] B. Nour et al., "A Unified Hybrid Information-Centric Naming Scheme for IoT Applications," Computer Communications, 2019.

[5] B. Nour et al., "Access Control Mechanisms in Named Data Networks: A Comprehensive Survey," ACM Computing Surveys, 2021.

[6] O. Serhane et al., "CnS: A Cache and Split Scheme for 5G-enabled ICN Networks," in IEEE ICC Conference, 2020.

[7] B. Nour et al., "A Survey of Internet of Things Communication using ICN: A Use Case Perspective," Computer Communications, 2019.

[8] B. Nour et al., "A Distributed ICN-based IoT Network Architecture: An Ambient Assisted Living Application Case Study," in IEEE GLOBECOM Conference, 2017.

[9] S. Muralidharan et al., "PPT: A Push Pull Traffic Algorithm to Improve QoS Provisioning in IoT-NDN Environment," IEEE Communications Letters, 2017.

[10] B. Nour et al., "A Collaborative Multi-Metric Interface Ranking Scheme for Named Data Networks," in IWCMC Conference, 2020.

[11] C. Fang et al., "A survey of energy-efficient caching in informationcentric networking," IEEE Communications Magazine, 2014.

[12] B. Nour et al., "A Distributed Cache Placement Scheme for Large-Scale ICN Networks," IEEE Network, 2020.

[13] M. Amadeo et al., "Information-centric networking for the internet of things: challenges and opportunities," IEEE Network, 2016.

[14] H. Asaeda et al., "CCNinfo: Discovering Content and Network Information in Content-Centric Networks," Internet Engineering Task Force, Internet-Draft, 2018.

[15] M. Meddeb et al., "How to cache in ICN-based IoT environments?" in IEEE/ACS AICCSA Conference, 2017.

[16] Y. Zou et al., "Energy-aware probabilistic forwarding in wireless content-centric networks," in ICTC Conference, 2016.

[17] S. Gao et al., "Energy efficient interest forwarding in NDN-based wireless sensor networks," Mobile Information Systems, vol. 2016, 2016.

[18] L. M. JSM et al., "Energy efficient context based forwarding strategy in named data networking of things," in ACM ICN Conference, 2016.

[19] X. Wang et al., "Energy-efficient ICN routing mechanism with QoS support," Computer Networks, 2018.

[20] O. Hahm et al., "A named data network approach to energy efficiency in IoT," in IEEE GLOBECOM Workshop, 2016.

[21] O. Serhane et al., "A Survey of ICN Content Naming and In-network Caching in 5G and Beyond Networks," IEEE Internet of Things Journal, 2020 . 